\title{
Infectious disease model with the latent period and quarantine and Comparison of drug distribution scheme based on Ebola virus
}

\author{
Jie Zhang \\ School of North China Electric Power University, Baoding 071000, China \\ 1213526050@163.com
}

Keywords: Infectious disease model; latent period; Ebola.

\begin{abstract}
Without considering the crowd with births and deaths, the model with the latent period and quarantine which based on the Ebola virus was established through using the method of dynamics. Compared the control effect of the outbreak under different medicine distribution scheme, and analyzed the key factors which may change the spread of the Ebola virus. This paper provide a theoretical basis for the prevention of infectious disease transmission.
\end{abstract}

\section{Introduction}

The current Ebola outbreak in West African countries may have been triggered by an initial infection in Dec 2013. The first case was thought to be a 2 years old patient lived in a village in Guinea. Ebola spreads through human-to-human transmission via direct contact with the blood, secretions, organs or other bodily fluids of infected people.

Since the 1920s, Kermark and Mckendrick primarily used the dynamics method to establish the mathematical model of infectious diseases, people gradually realized the significance of applying the theory and method of mathematical to explore infectious disease or general epidemiology. The existing infectious disease model was mostly established without considering the latency and quarantine. However, in reality, there is a latency after people is infected. This paper established an infectious disease model with the latent period and quarantine. For the sake of discussion, we do not consider the crowd of births and deaths.

\section{Organization of the Text}

\subsection{Ordinary Differential Equation Model for the transmission of Ebola.}

The transmission of infectious diseases like Ebola is often silent in the beginning, there could be a long time before people find it a disaster. During this period, the disease is spreading in a natural way. So we establish an ODE (Ordinary Differential Equation) model to describe the process.

We assume a fixed population of hosts that are numbered from 1 to $N$. At any given time $t$, host $i$ can be in one of the following states.

- S: Susceptible to infection.

- I : Infectious, that is, able to infect other hosts through direct contact.

- R: Removed, hosts who have died from the disease are in this state.

- U: Incubation hosts are in a periods before symptoms emerge, where they cannot infect other hosts.

The sets of hosts that are in states S, I, R, U will be referred to as the S-compartment [1], the I-compartment, the R-compartment, and the $\mathrm{U}$ compartment, and they are all a function of time $t$, respectively. Time in our models could be either continuous or discrete. Membership in the compartments vary from time to time.

Assumptions for the model.

1. The total population makes up an open system, people enter and leave the system at any possible time with rate $\mathrm{P} 1, \mathrm{P} 2$, respectively.

2. Hosts who are in incubation period cannot infect other hosts but they can become infectious at any time. 
3. Hosts who have died from Ebola are the removed.

4. Floating hosts belong to the S-compartment and the U-compartment.

5. We ignore demographics (births and deaths).

The Rate Equations.

As is shown in Fig. 1, the model [2] has four compartments, and therefore four equations are required to parameterize it.

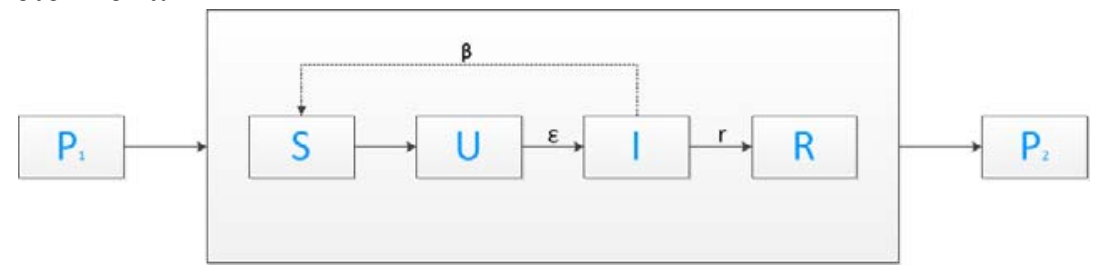

Fig. 1 An SUIR compartment model

The rate equations defining the model shown in Fig. 1 are listed below.

$$
\begin{aligned}
\frac{d S}{d t}=-\beta \frac{S}{N} I+P_{1}\left(1-\alpha_{1}\right) & -P_{2}\left(1-\alpha_{2}\right) \\
\frac{d U}{d t} & =\beta \frac{S}{N} I-\varepsilon U+\alpha_{1} P_{1}-\alpha_{2} P_{2} \\
\frac{d I}{d t} & =\varepsilon U-r I \\
\frac{d R}{d t} & =r I \\
\frac{d N}{d t} & =P_{1}-P_{2}
\end{aligned}
$$

Where

$\beta$ is the transmission rate;

$\mathrm{N}$ is the total population, and $\mathrm{S} / \mathrm{N}$ is the fraction of the population susceptible to infection;

$\mathrm{P} 1$ is the rate of hosts who enter the system;

$\mathrm{P} 2$ is the rate of hosts who leave the system;

$\alpha 1$ is the fraction of hosts who are in Incubation periods among people entering the system;

$\alpha 2$ is the fraction of hosts who are in in Incubation periods among people leaving the system;

$\varepsilon$ is a rate which reflects the incubation rate of Ebola;

$r$ is the death rate.

The incubation rate, recovery rate, and mortality can all vary based on socio-economic factors, gender, age, and infrastructure (health care, sanitation, water quality).

Rate Equations under Intervention.

The transmission of Ebola under medical intervention is different from its natural transmission mode. So we formulate another model to explore the transmission of Ebola under medical intervention. Based on the model we have established, the model includes more parameters to define complicated changes in the number of each compartment.

Added compartments:

- H: hosts who are in a clinical isolation.

- F: Ebola virus carrier, that is, able to infect the susceptible people.

- D: hosts who have died from Ebola and have been buried.

- E: hosts who are removed from the suspicious Infectious group list.

- G: hosts who have been considered infectious and received medical treatment.

- C: complete recovery.

The design of the model is shown in Fig. 2. The transmission pathway of Ebola is also captured in the model. 


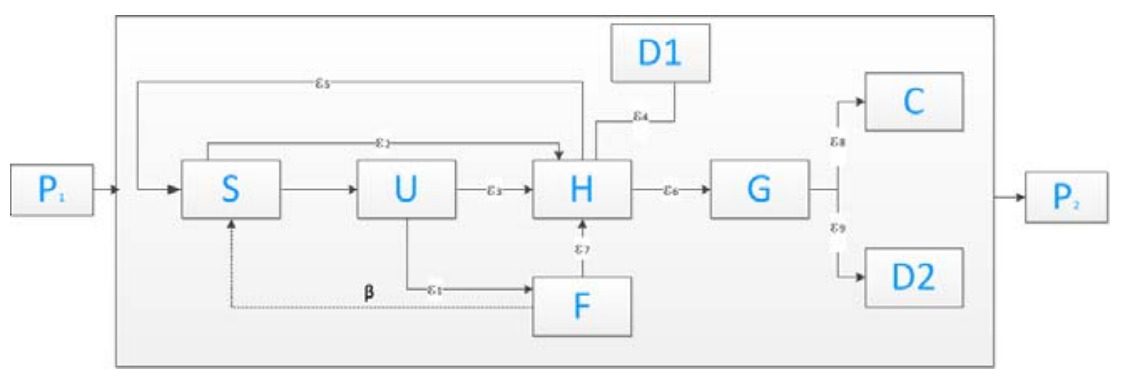

Fig. 2 A compartment model for Ebola

The rate equations defining the model shown in Fig. 2 are listed below.

$$
\begin{aligned}
& \frac{d S}{d t}=-\beta \frac{S}{N} F+P_{1}\left(1-\alpha_{1}\right)-P_{2}\left(1-\alpha_{2}\right)-\varepsilon_{2} S+\varepsilon_{6} H \\
& \frac{d U}{d t}=\beta \frac{S}{N} F-\varepsilon_{1} U+\alpha_{1} P_{1}-\alpha_{2} P_{2}-\varepsilon_{4} U \\
& \frac{d H}{d t}=\varepsilon_{3} U-\left(\varepsilon_{4}+\varepsilon_{5}+\varepsilon_{6}\right) H+\varepsilon_{7} F+\varepsilon_{2} S \\
& \frac{d G}{d t}=\varepsilon_{6} H-\left(\varepsilon_{8}+\varepsilon_{9}\right) G \\
& \frac{d F}{d t}=\varepsilon_{1} U-\varepsilon_{7} F \\
& \frac{d C}{d t}=\varepsilon_{8} G \\
& \frac{d D}{d t}=\varepsilon_{4} H+\varepsilon_{9} G
\end{aligned}
$$

We now solve the equation by Explicit Eulers Method [3, 4]. To simplify the problem, we tried setting the parameters, adjusted them for several times and found a best simulation result. The simulation result is shown in Fig. 3. We also searched Ebola 2014 parameters to compare with our simulation results in Fig. 4.

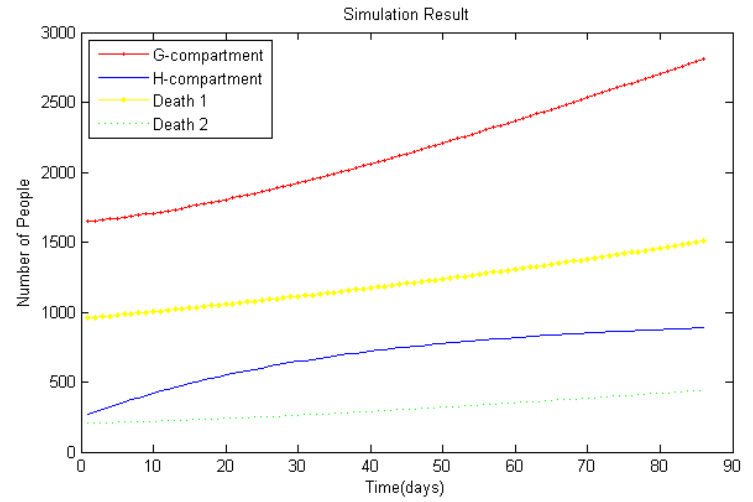

Fig. 3 Simulation results of the Ebola model

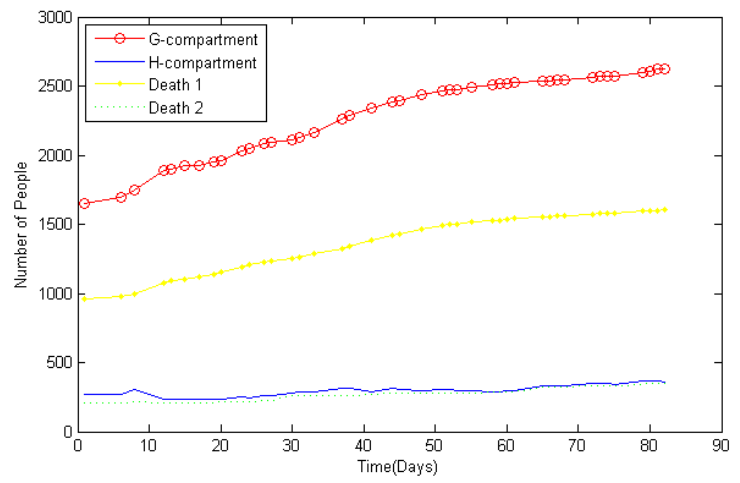

Fig. 4 Results based on real data Where

Death1 is the number of deaths who are confirmed;

Death 2 is the number of deaths who are probably infected.

\subsection{The Optimization of Medicine Assignment.}

To control the spread of Ebola, we decide to provide medicine for affected areas. Since infectious population is increasing rapidly and the speed of producing drugs is limited, so we have to optimize the assignment plans.

Assumptions:

- We have only two types of medicine: vaccines and drugs.

- The speed of manufacturing is a constant.

- Vaccines are very productive and work immediately.

The quantity of medicines is proportional to the number of people who need medical treatment, and limited by the productivity of medicine factories. So the quantity of medicines is a function of infected cases number and have an upper bound. We will estimate it based on actual data [5] later. 
We provide three plans for the assignment:

- The quantity of medicine assigned for areas are proportional to the population.

- Assign vaccines based on the number of H-compartment, and assign drugs based on the number of G-compartment.

- Assign vaccines based on the increased number of H-compartment in the following 85 days, and assign drugs based on the increased number of G-compartment in the following 85 days. Each of the plans is reasonable. We now formulate a function to define the effect of medicines. We consider the following 85 days after an area begin to use medicine, the number of the death and newly-confirmed in fectious patients is changing over time. Adapt three plans respectively, the best result comes when the total increased number is the smallest. Assume that we have two areas under consideration.

The function is given:

$W=\sum_{i=1}^{85} w_{i}$

Based on the function and our simulation results, we can compare three programs in Fig. 5.

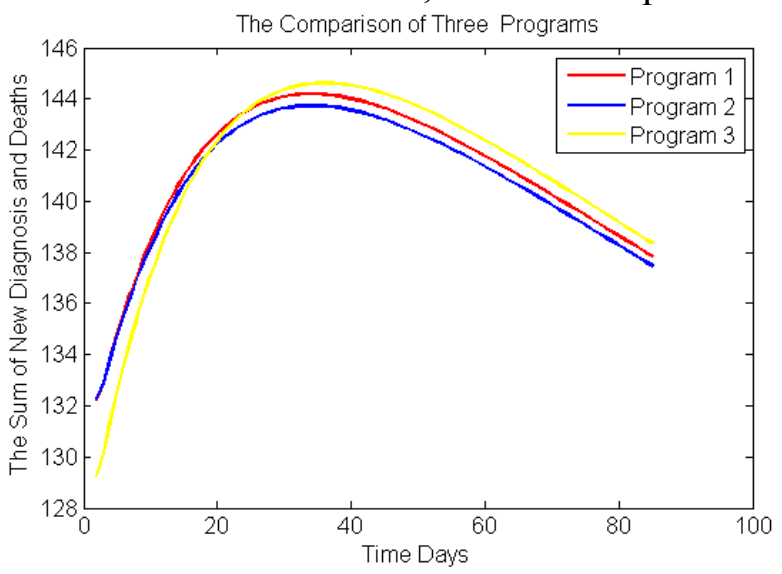

Fig. 5 Comparison of three programs

Apparently, Program 2 plays an important role in controlling the number of the infectious. Our assumption is proved reasonable.

\subsection{The effect of public awareness and border control.}

With the increase of the propaganda for Ebola, people would take some measures to prevent close contact with infectious person, such as avoid going to public place, wearing protection equipment etc. All of those changes would lead to one parameter change in our previously built model: the transmission rate $\beta$. If we reduce the transmission rate by 3 percent, the number of people in different state would be different. We use the parameters in model one as an example to show the difference. The result is shown in Fig. 6.

According to the previous assumption, we got that Floating hosts belong to the S-compartment and the U-compartment. Those U-compartment mingle with the S-compartment. So the border control is just to control the number of people who leave and enter this region. This leads change to the parameter $\mathrm{P} 1, \mathrm{P} 2$, if we switch $\mathrm{P} 1$ to $0.5 \mathrm{P} 1$, change $\mathrm{P} 2$ to $0.5 \mathrm{P} 2$. The result is shown in Fig. 7.

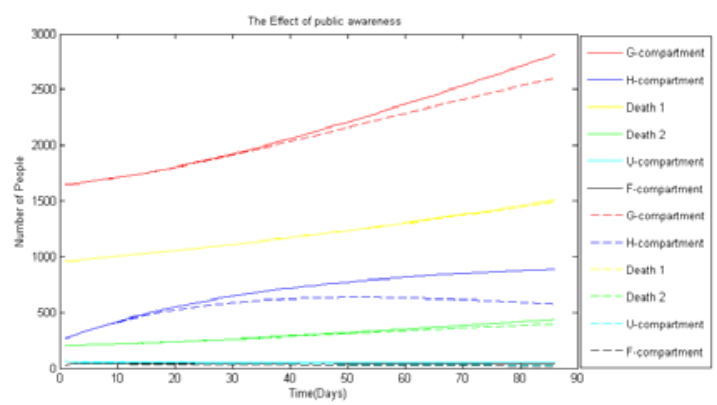

Fig. 6 The effect of public awareness

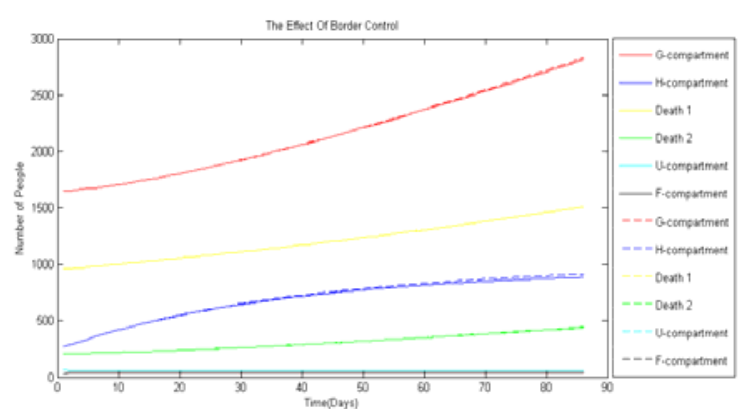

Fig. 7 The effect of border control

Conclusion 
We can see it clearly from Fig. 6 that the change lines growth rate of G-compartment is smaller, and the number of deaths is also smaller. The improvement of public awareness can restrain the spread of the Ebola.

From Fig. 7 we can see that the origin lines are quiet close to change lines. The effect is not obvious, this is mainly because that U-compartment in P1, P2 occupy little percentage. Border control does not make much difference to the spread of Ebola.

\section{Summary}

In this paper, compartment model was improved, considering the impact of latent period and quarantine. Through MATLAB simulation, it proves that the model fits very well with the actual data.

According to the simulation results of three medicine distribution scheme, the conclusion is as follow: the reasonable distribution of vaccines and drugs for different people can effectively control the spread of disease. At the same time, the measures to tackling the spread of infectious diseases should promptly be adopted (for example: public awareness and border control). It will play a significant role to control the epidemic situation of infectious diseases.

\section{References}

[1] Information on http://jsxgraph.uni-bayreuth.de/wiki/index.php/Epidemiology: The SIR model

[2] Information on https://wiki.eclipse.org/Introduction to Compartment Models

[3] Information on http://www.ohio.edu/people/just/IONTW/Models.pdf

[4] Information on http://en.wikipedia.org/wiki/Explicit and implicit methods

[5] Information on http://www.citypopulation.de/Guinea-Cities.html 